\title{
NK cells play a significant role in immunosurveillance at the early stage of MLL-AF9 acute myeloid leukemia via CD226/CD155 interactions
}

\author{
WANG YaJie ${ }^{1}$, CHEN Chen ${ }^{1}$, DONG Fang ${ }^{1}$, MA ShiHui ${ }^{1}$, XU Jing ${ }^{1}$, GONG YueMin ${ }^{1}$, \\ CHENG Hui ${ }^{1,2}$, ZHOU Yuan ${ }^{1}$, CHENG Tao ${ }^{1,2,3^{*}}$ \& HAO Sha ${ }^{1 *}$ \\ ${ }^{1}$ State Key Laboratory of Experimental Hematology, Institute of Hematology\& Blood Diseases Hospital, Collaborative Innovation Center for \\ Cancer Medicine, Tianjin 300020, China; \\ ${ }^{2}$ Center for Stem Cell Medicine, Chinese Academy of Medical Sciences \& Peking Union Medical College, Tianjin 300020, China; \\ ${ }^{3}$ Department of Stem Cell and Regenerative Medicine, Chinese Academy of Medical Sciences \& Peking Union Medical College, Tianjin \\ 300020, China
}

Received August 22, 2015; accepted September 16, 2015; published online November 19, 2015

\begin{abstract}
Acute myeloid leukemia (AML) is an aggressive hematological malignancy, and the mechanism underlying immune system involvement in leukemia development is unclear. In the present study, we utilized a myeloid/lymphoid or mixed-lineage leukemia; translocated to, 3 (MLLT3/MLL-AF9)-induced AML mouse model with or without exposure to irradiation. We found that the leukemia cells could survive and expand in hosts with intact immune systems, whereas leukemia progression was accelerated in mice with impaired immune systems. Moreover, the leukemia cells escaped from host immunosurveillance via editing their immunogenicity, including the up-regulation of an inhibitory antigen (i.e., CD47) and the down-regulation of active antigens (i.e., CD86, CD54, retinoic acid early transcript (RAE), histocompatibility 2, D region locus b (H2-Db) and H2-Dd). Natural killer (NK) cells were activated in the early phase of AML progression, whereas T cells were stimulated in the late phase. Furthermore, NK cell depletion showed that NK cells were necessary for the elimination of leukemia cells in our AML mouse model. Notably, CD155/CD226 primarily mediated the interaction between NK cells and leukemia cells and contributed to the antitumor effects of NK cells during the early phase of AML. Clinical data from patients with diverse hematological malignancies showed that CD155 expression was decreased in hematological malignancies. Taken together, our results demonstrate that NK cells play a pivotal role in immunosurveillance against leukemia cells during the early stage of AML primarily through the CD226/CD155 interaction; however, NK cells are not sufficient to eliminate leukemia cells.
\end{abstract}

acute myeloid leukemia, natural killer cell, immunosurveillance

Citation: Wang YJ, Chen C, Dong F, Ma SH, Xu J, Gong YM, Cheng H, Zhou Y, Cheng T, Hao S. NK cells play a significant role in immunosurveillance at the early stage of MLL-AF9 acute myeloid leukemia via CD226/CD155 interactions. Sci China Life Sci, 2015, 58: 1288-1298, doi: $10.1007 / \mathrm{s} 11427-015-4968-3$

Acute myeloid leukemia (AML) is a heterogeneous disorder characterized by the clonal expansion of distinct leukemia stem cells (LSCs) [1-3]. Although the majority of patients achieve complete remission with standard induction chemotherapy, the relapse rate remains high [4]. Allogeneic stem

*Corresponding author (email: chengtao@ihcams.ac.cn; haosha1012@163.com) cell transplantation is curative for some AML patients, possibly due to the therapeutic graft-versus leukemia effect [5]. Although only a minority of patients are suitable for transplantation, the success of this procedure has encouraged the investigation of other immune strategies against leukemia [6-8].

Leukemia-associated antigens (LAA) are expressed on 
leukemia progenitors [9]. Some LAAs, such as proteinase 3 (PR3) and Wilm's tumor antigen 1 (WT1), elicit a cytotoxic T-cell response [10]. Moreover, AML cells employ immune evasion strategies to escape immunosurveillance (i.e., the up-regulation of calreticulin/cluster of differentiation 47 (CD47) [11], programmed death-ligand 1 (PD-L1)/CD274) [12] and toll-like receptors [13]). Notably, clinical data based on strategies targeting these antigens are diverse, suggesting that leukemia cells may evolve gradually and exhibit interplay with the immune system during leukemia progression.

The immune system not only eliminates developing leukemia but also sculpts the immunogenicity of leukemia cells. Modified leukemia cells could survive in immunocompetent hosts. The adaptive immune response dominated by $\mathrm{T}$ cells has been demonstrated to be an essential component of cancer immunosurveillance $[14,15]$. Natural killer (NK) cells play a key role in the immune antitumor response, as demonstrated by the low relapse rate obtained for allogenic stem cell transplantation when there is a mismatch in NK inhibitory receptors between the host and the graft [16]. Interestingly, recent studies reported that AML blasts induced long-lasting changes in NK cells (i.e., impairing their effect or function and reducing the competence of the innate immune system), thereby favoring leukemia survival $[17,18]$. Moreover, NK cells in AML patients showed diminished expression of several activating receptors that contributed to impaired NK cell function, and as a consequence AML blasts escaped from NK cell immunosurveillance [19]. However, the mechanism by which leukemia cells survive in immunocompetent hosts and what role NK cells precisely play during leukemia development in a more clinically relevant setting are unclear.

In this study, we used a previously reported AML mouse model (MLL-AF9-induced AML) [20,21]. Established MLL-AF9 leukemia cells were transplanted into immunocompetent mice with or without irradiation. We found that the destruction of the immune system by irradiation accelerated the progression of leukemia. Established leukemia cells have evolved by modifying antigens to escape immunosurveillance, especially compared with hematopoietic stem progenitor cells (HSPCs). Nevertheless, modified leukemia cells could still activate innate and adaptive immune responses during different phases of the disease. Leukemia development was largely inhibited by NK cells even in the absence of adaptive immunity. NK cells were mainly activated by the upregulation of activating ligands (i.e., CD155 and RAE on leukemia cells) during the early phase of the disease. Notably, the activated ligands were down regulated in LSCs, which occupy the bone marrow (BM) pool during the late phase. Clinical data from patients with diverse hematological malignancies also showed reduced CD155 expression. These results indicate that NK cells play a vital role in the antitumor response primarily during the early phase of MLL-AF9 AML. Furthermore, leukemia cells modify their immunogenicity to generate a repertoire that is capable of survival in immunocompetent hosts.

\section{Materials and methods}

\subsection{In vivo mouse model}

C57BL/6-CD45.2 and Nod-scid mice between 6-8 weeks of age were maintained in the animal facility of the State Key Institute of Hematology (SKLEH). All experiments were conducted under the protocol approved by the Institutional Animal Care and Use Committees of SKLEH. Lin ${ }^{-}$stem and progenitor cells were enriched from the bone marrow cells of C57BL/6-CD45.2 mice using lineage cell depletion beads. Enriched $\mathrm{Lin}^{-}$cells were infected with a retrovirus carrying MSCV-MLL-AF9-IRES-GFP (green fluorescent protein) as previously described. After $2 \mathrm{~d}$ of transduction, approximately $1 \times 10^{6}$ cells were injected into the lethally irradiated (9.5 Gy) C57BL/6-CD45.2 recipients. The dosage was split into 4.75 Gy twice a day. Mice were sacrificed at the terminal stage of leukemia ( $60 \mathrm{~d})$, and primary leukemic cells $(\mathrm{P} 0)$ in the bone marrow were harvested for the in vivo experiments.

\subsection{Sample preparation}

Lin $^{-}$cells were isolated using a negative magnetic bead selection protocol (Miltenyi, Bergisch Gladbach, Germany). $\mathrm{CD}^{+} \mathrm{T}$ cells, $\mathrm{CD}^{+} \mathrm{T}$ cells and NK cells were sorted by FACS (Becton Dickinson, Franklin Lakes, USA). T cells were cultured in Roswell Park Memorial Institute (RPMI) 1640 medium supplemented with $10 \%$ fetal bovine serum (FBS) and IL-2 (20 ng mL $\left.{ }^{-1}\right)$.

\subsection{Flow cytometry}

A total of $5 \times 10^{6}-1 \times 10^{7}$ harvested cells were suspended in $100 \mu \mathrm{L}$ of FACS buffer and stained with the corresponding antibodies at $4{ }^{\circ} \mathrm{C}$ for the appropriate amount of time. Anti-CD3, anti-CD4, anti-CD8, anti-CD45R, anti-CD11b, anti-Gr-1, and anti-Ter-119 constituted the Lineage mixture (Lin mix). The following antibodies were used to study HSCs and HPCs: Lin mix, anti-c-Kit, anti-Sca1, anti-CD34, anti-CD150, anti-CD16/32 (Fc $\gamma$ R), anti-CD135 (Flt3/Flk2), anti-CD45.1 and anti-CD45.2 (BD Biosciences). Anti-Mac1 and anti-Gr1 were used to distinguish the myeloid lineage. Anti-CD3, anti-CD4 and anti-CD8 were used to study T cells, whereas anti-NK1.1 was used to analyze NK cells. Leukemic cells could be distinguished by reading the fluorescence of the GFP marker. 4',6-diamidino-2-phenylindole (DAPI) was applied to distinguish dead cells. Cell analyses were performed on the LSRII flow cytometer (Becton Dickinson, Franklin Lakes, USA). Cell sorting assays were performed on the AriaIII flow cytometer (Becton Dickinson, Franklin Lakes, USA). 


\subsection{NK cell depletion}

The C57BL/6 and Nod-scid mice were irradiated with 4.5 and $2 \mathrm{~Gy}$, respectively, and intraperitoneally injected with an anti-CD122 $(100 \mu \mathrm{g})$ antibody $1 \mathrm{~d}$ post-irradiation. Two days later, NK cell depletion in the BM and spleen was confirmed by flow cytometry.

\subsection{CFSE staining}

Single-cell suspensions of $\mathrm{T}$ cells were resuspended at a density of $5-10 \times 10^{6}$ per $\mathrm{mL}$ in PBS (pre-warmed to room temperature). CFDA SE; 5, 6-carboxyfluorescein diacetate, succinimidyl ester (CFSE) was added to the desired final concentration $\left(1 \mu \mathrm{mol} \mathrm{L} \mathrm{L}^{-1}\right)$, mixed immediately and incubated for $10 \mathrm{~min}$ at room temperature in the dark. The labeling was stopped by the addition of 4-5 volumes of cold complete medium, and the cells were incubated on ice for $5 \mathrm{~min}$. The cells were washed 3 times with complete medium, and the T cells were cultured in RPMI 1640 with $10 \%$ FBS.

\subsection{Quantitative RT-PCR (qRT-PCR)}

Total RNA was isolated using the Qiagen RNeasy mini kit. qRT-PCR assays were performed on the StepOne real-time PCR system (Applied Biosystems).

\subsection{Statistical analysis}

The GraphPad Prism 5.0 and SPSS 17.0 statistical software were used for statistical analysis. An unpaired Student's $t$ test and analysis of variance (ANOVA) were applied to generate $P$ values for most datasets.

\section{Results}

\subsection{Destruction of the immune system accelerates leu- kemia progression}

To study the interplay between leukemia cells and the immune system, we used our previously reported leukemia mouse model [20,21]. Lin ${ }^{-}$BM HSPCs were purified from mice and transfected with the MSCV-MLL-AF9-IRES-GFP retrovirus as previously described. Transformed cells were transplanted into lethally irradiated mice. The recipients developed leukemia within 2 months (Figure 1A). The established leukemia cells were positive for GFP and myeloid lineage markers, which indicated an AML phenotype. The primary leukemic cells were able to induce AML when injected into secondary recipients, demonstrating the transplantability of the leukemic cells in our system. Then, the established leukemic cells could be used to perform the subsequent experiments.

Irradiation has been reported to damage the immune system [22]. To determine whether the breakdown occurred in our experiment system, two irradiation doses (4.5 and $6 \mathrm{~Gy})$ were chosen. NK cells and T cells were selected as the representatives for innate immunity and adaptive immunity, respectively. There was no significant difference in $\mathrm{T}$ cells in the BM between the irradiated group and the non-irradiated group. In contrast, $\mathrm{T}$ cells were obviously decreased in the spleen, peripheral blood (PB) and thymus of the irradiated group (Figure 1B and C). Notably, NK cells were significantly reduced in the $\mathrm{BM}$, spleen and $\mathrm{PB}$ of the irradiated mice (Figure 1B and C). These phenomena suggest that the irradiation causes damage to the mouse immune system as expected.

Next, we examined whether the immune system participated in leukemia progression. A total of $5 \times 10^{4}$ leukemic cells were transplanted into irradiated or non-irradiated mice. The irradiated mice treated with a dose of either 4.5 or $6 \mathrm{~Gy}$ showed a shorter lifespan compared with the non-irradiated mice (Figure 1D). These results demonstrated that the destruction of the immune system by irradiation could accelerate the progression of AML. Surprisingly, in contrast to a previous report using a myeloid/lymphoid or mixed-lineage leukemia; translocated to, 1 (MLLT1/ MLL-ENL) leukemia model [23], the non-irradiated recipients in our model succumbed to AML despite their intact immune systems. This suggests that the immunosurveillance effect of the immune system during the development of MLL-AF9 AML is limited.

\subsection{Modified immunogenicity of leukemic cells is in- volved in leukemia initiation}

The leukemic cells could survive in immunocompetent mice and cause a leukemia outbreak. Therefore, we hypothesized that the immunogenicity of leukemic cells could be altered by MLL-AF9, especially compared with normal HSPCs, because some antigen molecules have been reported to be dominantly expressed on malignant cells. First, we evaluated the expression of the inhibitory antigens CD47 and CD274. We found higher expression of CD47 in the leukemic cells, but no distinction was found when CD274 expression was compared with HSPCs. Then, we analyzed the expression of the activating ligands CD86 [24] and MHCII and found a 50\% down-regulation of CD86. CD54 [25] was also reduced in leukemic cells, which was similar to findings reported for patients with lymphoproliferative disorders [26]. We also detected some ligands of activated receptors on NK cells, which could enhance NK-mediated cytotoxicity. Among these ligands, CD155 exhibited increased expression on leukemic cells compared with HSPCs, whereas RAE and H2-Dd had reduced expression. These changes in leukemic cell antigens may imply the modification of the immunogenicity of leukemic cells is in concurrence with leukemia progression (Figure 2A and B).

\subsection{T cells and NK cells are activated during different stages of leukemia progression}

To investigate the interplay between leukemic cells and the 

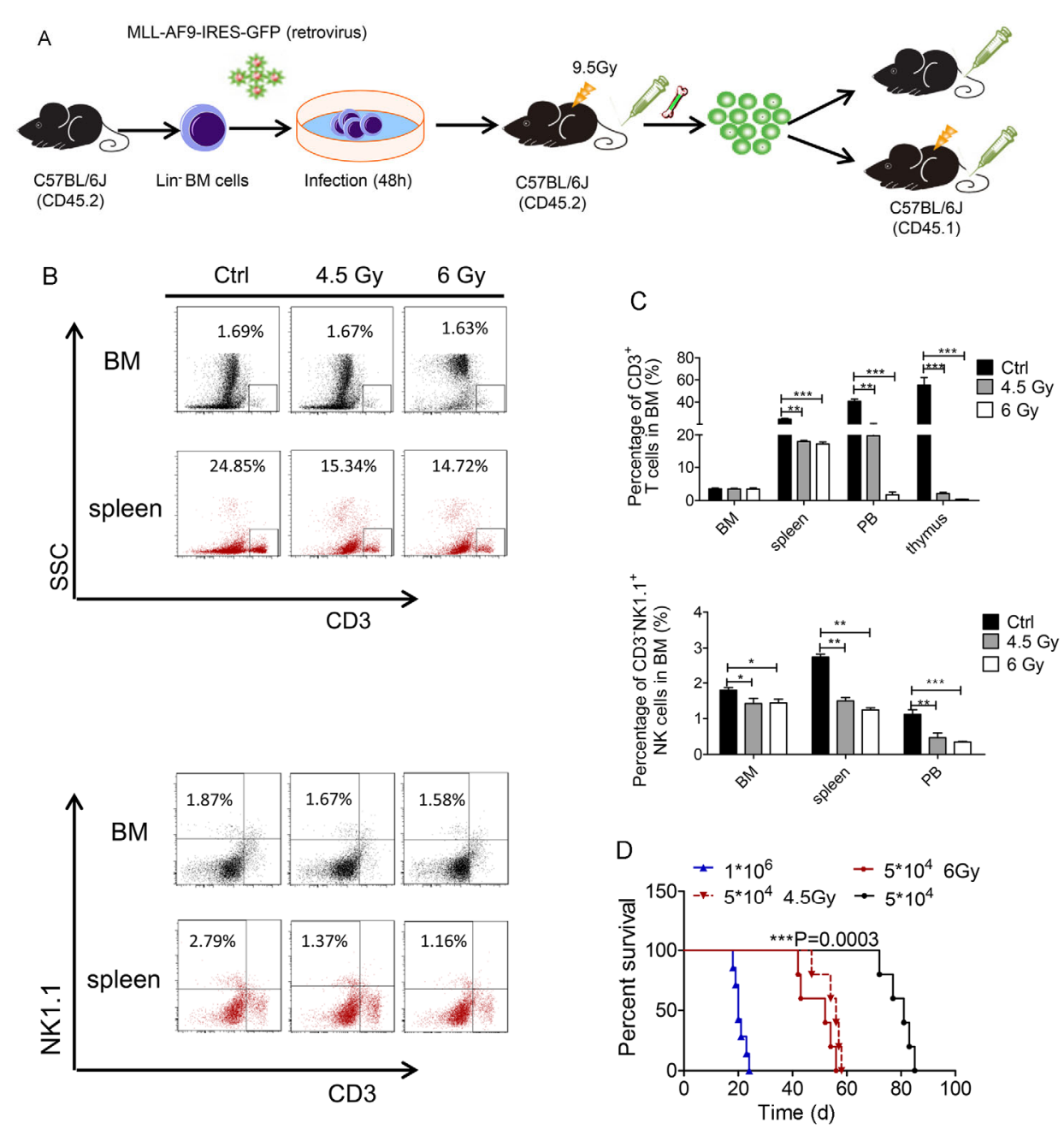

Figure 1 Immune cells play a large role in anti-acute myeloid leukemia. A, Strategy used to establish MLL-AF9 leukemic mice. BM Lin ${ }^{-}$cells were transduced with MSCV-MLL-AF9-IRES GFP for $48 \mathrm{~h}$ and then transplanted in lethally irradiated mice (9.5 Gy). Established MLL-AF9 leukemic cells were transplanted in recipient mice in subsequent experiments. B, Representative flow graphs showing the rates of T cells and NK cells in the BM and spleen of recipient mice 3 days post-irradiation. C, Percentages of T cells (upper panel) and NK cells (lower panel) in the recipient mice 3 days post-irradiation. Data represent the mean $\pm \mathrm{SD}$ ( $n=3$ per group, 3 independent experiments). ${ }^{*}: P<0.05 ;{ }^{* *}: P<0.001$, one-way ANOVA analyses. D, Kaplan-Meier curves of the overall survival of irradiated or non-irradiated wild-type mice transplanted with MLL-AF9 leukemia cells $(n=6)$.

host immune system, we used immunocompetent mice transplanted with $1 \times 10^{6}$ established leukemic cells for the following experiments. The manageable and suitable leukemia duration was performed as previously described. After transplantation with leukemic cells, the mice showed some fluctuation of immune cells. The percentage of T cells was obviously increased during the late stage ( day 20), whereas the percentage of NK cells gradually decreased. However, the total numbers of both cell types were reduced, especially during the late phase of leukemia (Figure $2 \mathrm{C}$ and D).

Next, we investigated whether the changes in the immune cells in leukemic mice had an antitumor effect in our model. Because $\mathrm{T}$ cells play a significant antitumor role, we sorted the $\mathrm{GFP}^{-} \mathrm{CD}^{+} \mathrm{CD}^{+} \mathrm{T}$ cells and $\mathrm{GFP}^{-} \mathrm{CD}^{+} \mathrm{CD}^{+} \mathrm{T}$ cells for mRNA analysis. The qRT-PCR results showed that transcription factors such as T-bet, RoR $\gamma$ t, Foxp3 and Gata3 were gradually upregulated in $\mathrm{CD}^{+} \mathrm{CD}^{+} \mathrm{T}$ cells concomitant with leukemia progression, especially during the late phase (day 14) of leukemia (Figure 3A). Cytotoxic molecules, including IFN- $\gamma$, granzyme and perforin, showed higher expression levels in $\mathrm{CD}^{+} \mathrm{CD}^{+} \mathrm{T}$ cells during the late stage (Figure 3B). To determine the activation of $\mathrm{T}$ cells in leukemic mice, we purified $\mathrm{CD}^{+} \mathrm{CD}^{+}$and $\mathrm{CD}^{+} \mathrm{CD}^{+} \mathrm{T}$ cells on day 14 and analyzed their proliferation by CFSE staining (Figure 3C). In line with the in vivo gene expression analyses, the percentage of dividing cells (\% Divided) and average number of cell divisions (Div.Index) of $\mathrm{T}$ cells in mice with leukemia were significantly upregulated after $48 \mathrm{~h}$ of culture in vitro (Figure 3D). In contrast, the cytotoxic molecules of NK cells (i.e., IFN- $\gamma$, granzyme and perforin) were activated during the early stage (day 7) but were markedly reduced to the basal level during the late stage (Figure $3 \mathrm{E}$ ). These results suggest that 

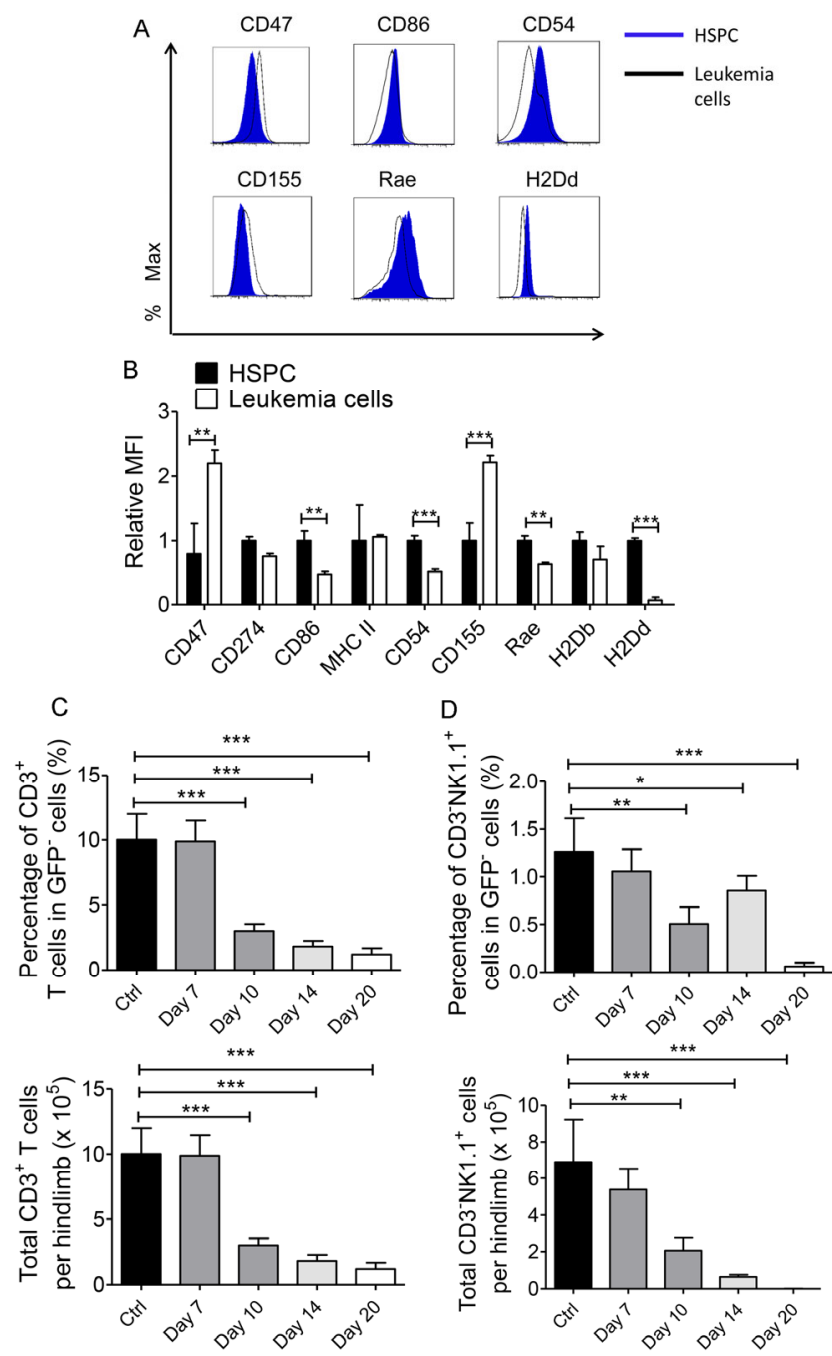

Figure 2 Change of the immune system during leukemia progression. A, Representative flow graphs showing the variation in immune antigen expression between LSK (HSPCs) and established leukemic cells. B, Histograms showing the MFI of different immune antigens ( $n=3$ per group, 3 independent experiments). C and D, Histograms showing the statistical analysis of the percentages and absolute numbers of T cells (C) and NK cells (D) of mice at the indicated time points after the induction of leukemia. Data represent the mean \pm SD $(n=6$ per group, 3 independent experiments). ${ }^{* *}: P<0.01 ;{ }^{* *}: P<0.001$, one-way ANOVA analyses.

T cells and NK cells are activated during the late stage and the early stage of the leukemia course, respectively, but these cells are not sufficient to eliminate the leukemic cells. Thus, we can infer that even the undamaged immune system cannot achieve the clearance of the evolving leukemic cells.

\subsection{NK cells play a vital but limited role in the fight against leukemia progression}

To determine the mechanism by which the activated immune cells were involved in the progression of leukemia, MLL-AF9 leukemic cells were intravenously transferred into wild-type and Nod-scid (lacking T cells, B cells and
NKT cells) mice. There were no significant differences in the survival curves of the Nod-scid and wild-type mice (Figure 4A). Next, to investigate the precise role of NK cells in AML, we used an anti-CD122 antibody to eliminate NK cells in both the wild-type and Nod-scid mice [27,28]. $\mathrm{NK}$ cells in the BM and spleen were significantly reduced after treatment with the anti-CD122 antibody (Figure 4B and C). Strikingly, NK cell-depleted WT mice showed accelerated leukemia development (median $4 \mathrm{~d}$ less than the control) (Figure 4D), whereas the NK cell-depleted Nod-scid recipients showed a significantly increased acceleration of leukemia (median $8 \mathrm{~d}$ less than the control) (Figure 4E). These results suggest that NK cells play a significant but not sufficient role in the elimination of leukemic cells. Thus, $\mathrm{T}$ cells are involved in the immunosurveillance of leukemia primarily through cooperation with NK cells.

\subsection{CD155/CD226 mainly mediates the interaction between NK cells and leukemic cells in AML}

To investigate the interaction between leukemic cells and NK cells, representative NK cell receptors and their specific ligands were analyzed during leukemia progression [29]. Consistent with the results of a previous study, we found the inhibition of natural killer group 2 membrane D (NKG2D) receptor [30,31] in NK cells but the upregulation of its ligand RAE in the late phase. These results confirmed that the NKG2D/RAE interaction was involved in the immunosurveillance activity of NK cells in AML (Figure 5A and C). Importantly, we found a gradual downregulation of DNAM-1 (CD226) in NK cells from leukemic mice at day 10 (Figure 5A) and an early upregulation (day 7) and late (day 10 and 20) downregulation of its ligand CD155 (Figure $5 \mathrm{C})$. Together with the changes of gene expression in NK cells (Figure 2E), these results indicate that the CD226/CD155 interaction plays a vital role in the NK cell-mediated suppression of leukemia. Additionally, we also found the upregulation of MHC class I-H2Db, which may mediate resistance of the leukemic cells to NK cell killing (Figure 5C). In contrast, no significant difference or fluctuation was detected in the two other crucial active and inhibitory receptors of NK cells (i.e., NKP46 and NKG2A) (Figure 5B).

Next, we investigated the effect of NK cells the immunogenicity of leukemic cells. The changes in leukemic cell antigens were reanalyzed in NK cell-depleted mice. The median fluorescence intensity (MFI) of MHC class $\mathrm{I}-\mathrm{H} 2 \mathrm{Dd} / \mathrm{H} 2 \mathrm{Db}$ of leukemic cells showed inconsistent fluctuation in the BM and spleen, whereas both CD155 and RAE of leukemic cells were upregulated in either the BM or spleen after NK cell depletion (Figure 5D and E). These results suggest that some leukemic cells down-regulate their active ligands for NK cells to escape the attack by NK cells. NK cells may kill leukemic cells that express higher levels of active signals. 
A
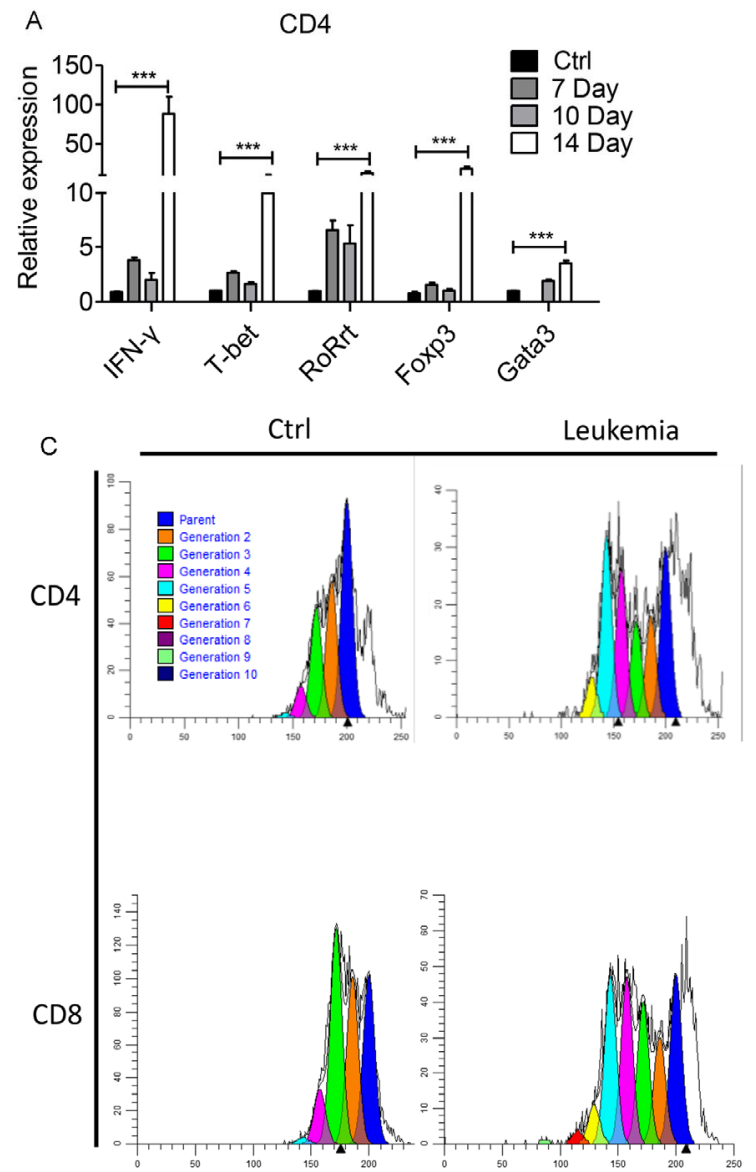

$\mathrm{E}$
B

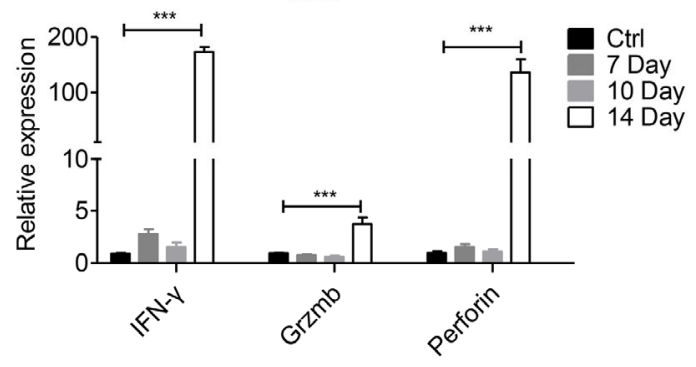

D
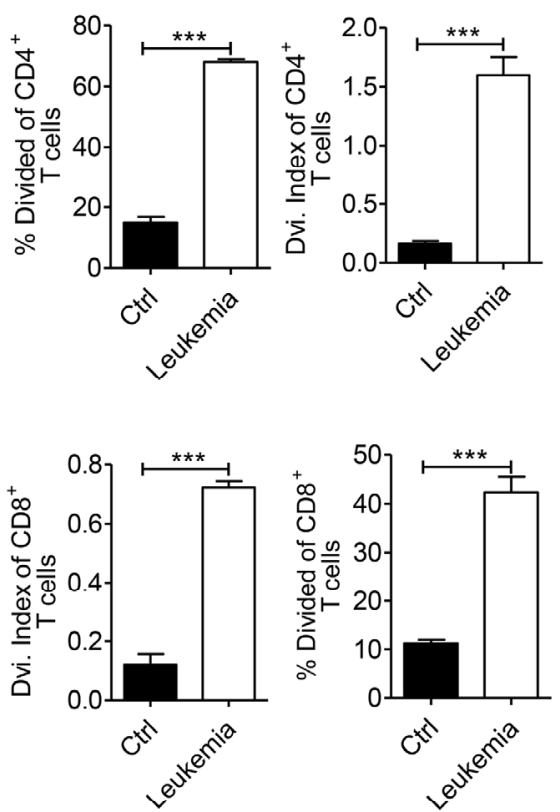

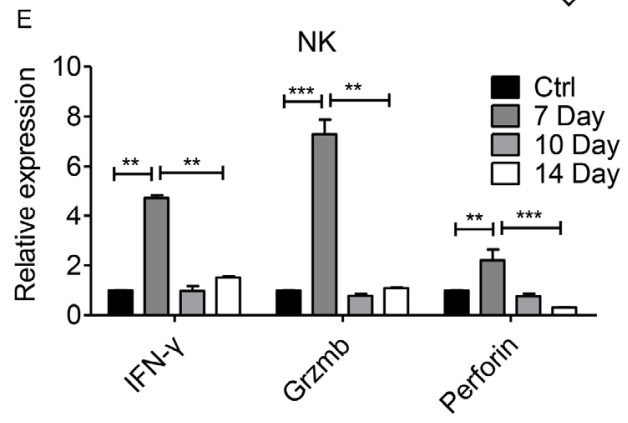

Figure 3 Activated immune system in leukemic mice. A and B, qRT-PCR analysis showing the expression levels of candidate genes in $\mathrm{GFP}^{-} \mathrm{CD} 3^{+} \mathrm{CD} 4^{+}$ (A) and $\mathrm{GFP}^{-} \mathrm{CD}^{+} \mathrm{CD}^{+}$(B) T cells at the indicated time points after leukemia cell transplantation. Data represent the mean $\pm \mathrm{SD}(n=6,3$ independent experiments). ${ }^{*}: P<0.05 ;{ }^{* *}: P<0.01 ;{ }^{* *}: P<0.001$, Student's $t$-test. C, Representative flow cytometry showing the proliferation of T cells based on CFSE staining of control and $14 \mathrm{~d}$ leukemic mice after culture for $48 \mathrm{~h}$. D, The histograms show the percentages of dividing cells (\% Divided) and the average number of cell divisions (Div. Index) of $\mathrm{GFP}^{-} \mathrm{CD}^{+} \mathrm{CD}^{+}$and $\mathrm{GFP}^{-} \mathrm{CD}^{+} \mathrm{CD}^{+}$cells. Data represent the mean $\pm \mathrm{SD}\left(n=3,2\right.$ independent experiments). ${ }^{* *}$ : $P<0.01 ;{ }^{* \star *}: P<0.001$, Student's $t$-test. E, qRT-PCR analysis showing the expression levels of candidate genes in $\mathrm{GFP}^{-} \mathrm{CD} 3^{-} \mathrm{NK} 1.1^{+} \mathrm{NK}$ cells.

2.6 Active molecules of NK cells are selectively inhibited in both LSCs and human hematological malignancies

To confirm the effects of active molecules of NK cells on the evolution of leukemic cells, we analyzed the expression of the main factors reported in previous studies [29]. The results showed that most molecules were reduced in AML
LSCs (with the exception of CEACAM1) based on the unsupervised clustering analysis (Figure 6A). Reduced expression of RAE, CD72 and CD155 on LSCs was observed compared to HSCs. Next, we analyzed the immunogenicity of hematological malignant cells in human samples using Oncomine analysis. Notably, the expression of CD155 was decreased in leukemia as well as lymphoma and myeloma 

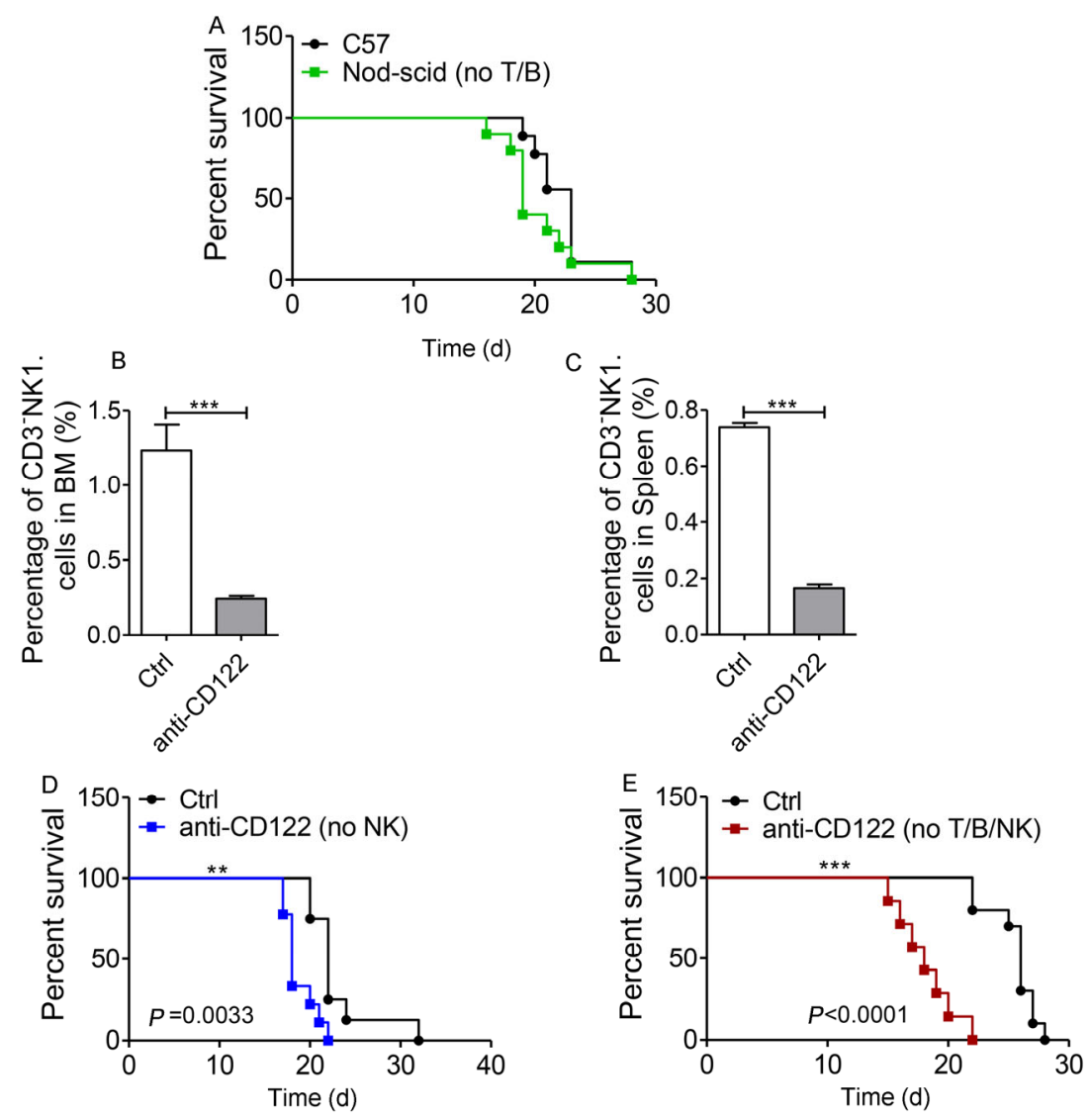

Figure 4 NK cells inhibit MLL-AF9 leukemia development even in the absence of adaptive immunity. A, Kaplan-Meier curves for the overall survival of C57BL/6-CD45.2 mice or Nod-scid mice transplanted with $5 \times 10^{5}$ MLL-AF9 leukemic cells ( $n=10$ per group). B and C, The histograms show the efficiency of NK cell depletion in the BM and spleen by the anti-CD122 antibody ( $n=3,2$ independent experiments). ${ }^{* * *}: P<0.001$, Student's $t$-test. D, Kaplan-Meier curves for the overall survival of sublethally irradiated (4.5 Gy) C57BL/6-CD45.2 wild type mice or NK-depleted (anti-CD122) mice transplanted with $1 \times 10^{5}$ MLL-AF9 leukemic cells ( $n=10$ per group). E, Kaplan-Meier curves for the overall survival of sublethally irradiated ( 2 Gy) Nod-scid wild type mice or NK-depleted (anti-CD122) mice transplanted with $1 \times 10^{5}$ MLL-AF9 leukemic cells ( $n=10$ per group).

based on COPA analysis. Moreover, RAE was upregulated, and distinct fluctuations of H2-Dd were shown in hematological malignancies (Figure 6B). These results indicate that the survival of leukemic cells may be partially attributed to the low expression levels of active ligands for NK cells (i.e., CD155) in mice and humans.

\section{Disscusion}

Investigation into the interplay between the immunoediting of leukemic cells and the immune system is necessary for the development of novel agents that target leukemic cells and cooperate with conventional treatments. In the present study, we employed a previously reported MLL-AF9 leukemic cell model to evaluate the interactions between leukemic cells and the immune system (Figure 1A). We found more aggressive leukemia progression in mice with deficient immune systems (Figure 1B-D). Moreover, the leukemic cells established by MLL-AF9 could survive and expand in robust hosts with an intact immune system, pos- sibly due to their modified immunogenicity. We summarized the upregulation of CD47 and CD155 and the downregulation of active antigens (i.e., CD86, CD54, RAE, $\mathrm{H} 2 \mathrm{Db}$ and $\mathrm{H} 2-\mathrm{Dd}$ ) in leukemic cells compared with HSPCs (Figure 2A). NK cells were activated in the early phase and $\mathrm{T}$ cells were stimulated in the late phase of the disease to fight against the immuno-edited leukemic cells (Figure 3A-E). Nevertheless, NK cells played a more significant role in the anti-leukemia response in AML, although they still exhibited some cooperative crosstalk with the adaptive immune system (Figure 4A-E). Additionally, we found that the anti-leukemia effect in AML mediated by NK cells mainly depended on the CD226/CD155 interaction (Figure $5 \mathrm{~A}-\mathrm{E}$ ). Importantly, some active antigens (i.e., CD155 for NK cells) showed reduced expression in LSCs and evolved leukemic cells from humans. In conclusion, NK cells play a significant role in immunosurveillance during the early phase of AML. NK cells may also participate in cooperative crosstalk with $\mathrm{T}$ cells to operate an immune response against leukemic cells though a mechanism that has yet to be defined (Figure 6C). 

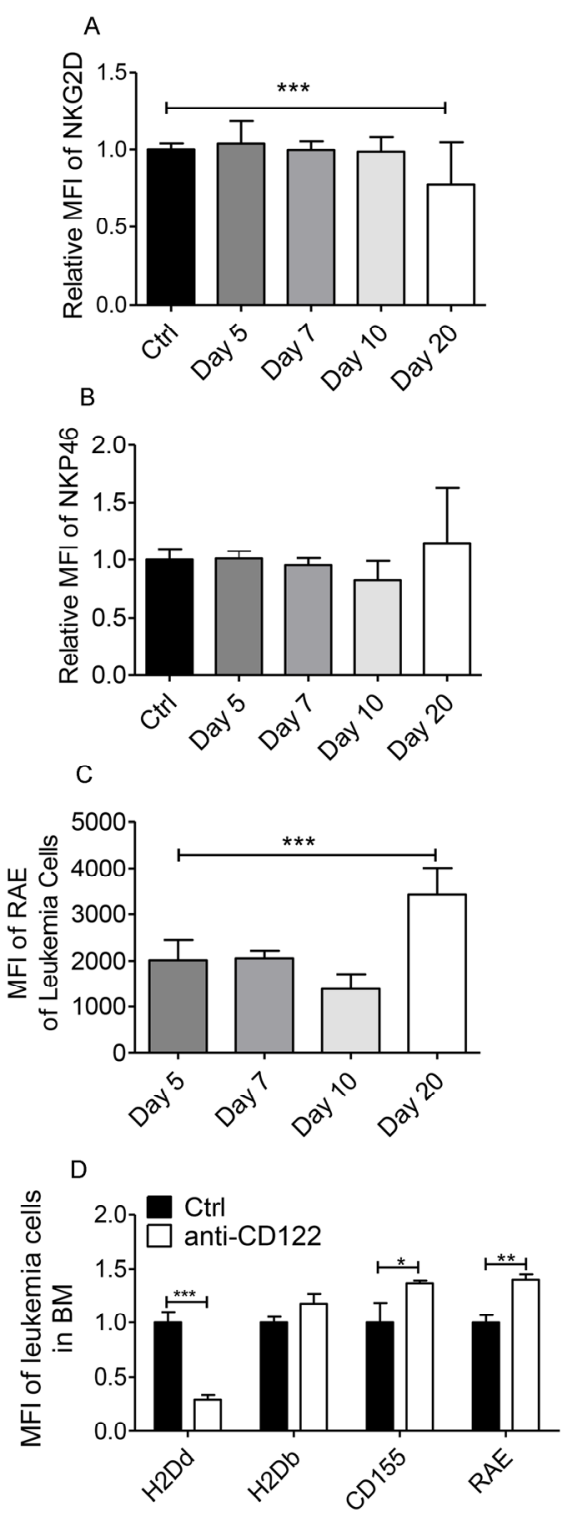
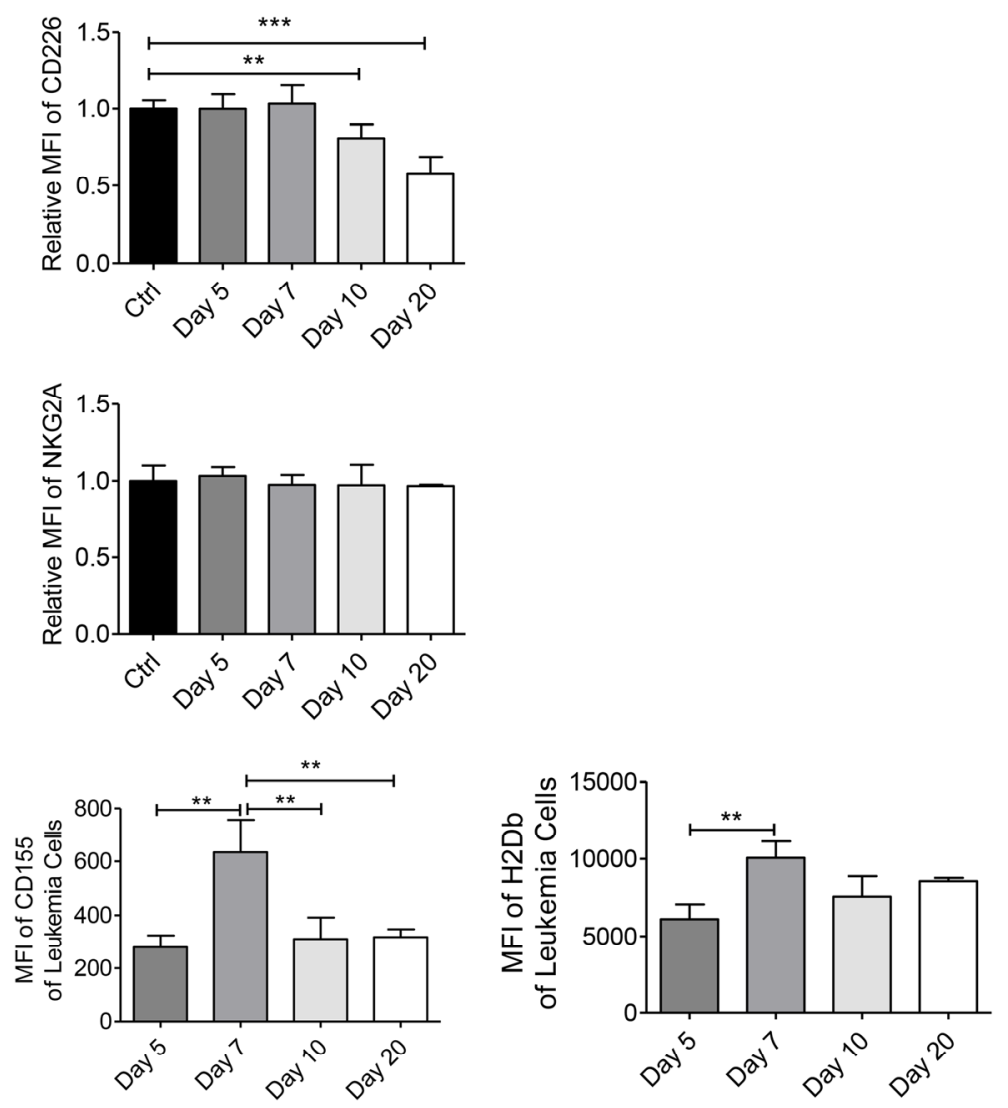

E

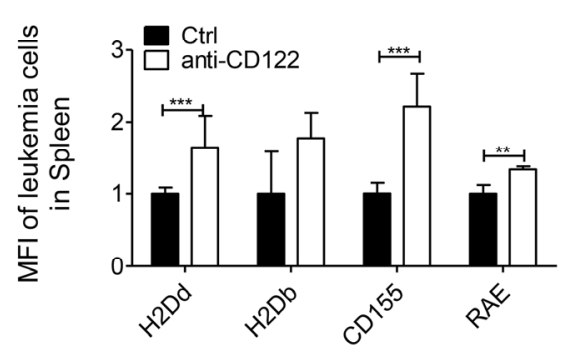

Figure 5 Expression of NK cell-activating ligands on leukemic cells. A and B, The histograms show the MFI of NK cell receptors at different post-leukemia time points ( $n=3,3$ independent experiments). ${ }^{* * *}: P<0.001$, Student's $t$-test. C, The histograms show the MFI of NK cell ligands on leukemic cells at different post-leukemia time points $\left(n=3,3\right.$ independent experiments). ${ }^{* \star}: P<0.001$, Student's $t$-test. D and E, The histograms show the variation of NK cell ligands on leukemic cells in wild type and NK cell-depleted mice ( $n=3,3$ independent experiments). ${ }^{* * *}: P<0.001$, Student's $t$-test.

In line with previous studies, established leukemic cells expressed higher inhibitory antigens and lower active ligands to escape immune attack, especially compared with HSPCs (Figure 2A). Additionally, leukemic cells exhibited CD155 upregulation and could activate NK cells during the early phase. J Nakata previously reported that the MLLENL oncogene could transform non-immunogenic hematopoietic cells into immunogenic cells that could be wholly eliminated [23]. Perhaps due to the different oncogenes and methods used, in our study the transplantation of leukemic cells ultimately led to animal death. Notably, we dynamically monitored the immunogenicity of leukemic cells during the progression of leukemia and observed the downreg- ulation of CD155 and upregulation of RAE. Because clinically evident leukemic cells may have been immunologically selected, they cannot fully reflect the integrated process of leukemia progression [32]. We demonstrated the immunogenic fluctuations of established leukemic cells during disease progression, which may offer some insight into the evolution of leukemic cells.

NK cell ligands were regarded as the intrinsic sensors of tumor transformation and an induced immune response $[33,34]$. NK cells were demonstrated to kill tumor cells due to the enforced expression of NK cell-activating ligands. These findings suggest that NK cells have the potential to recognize and eliminate developing tumor cells 


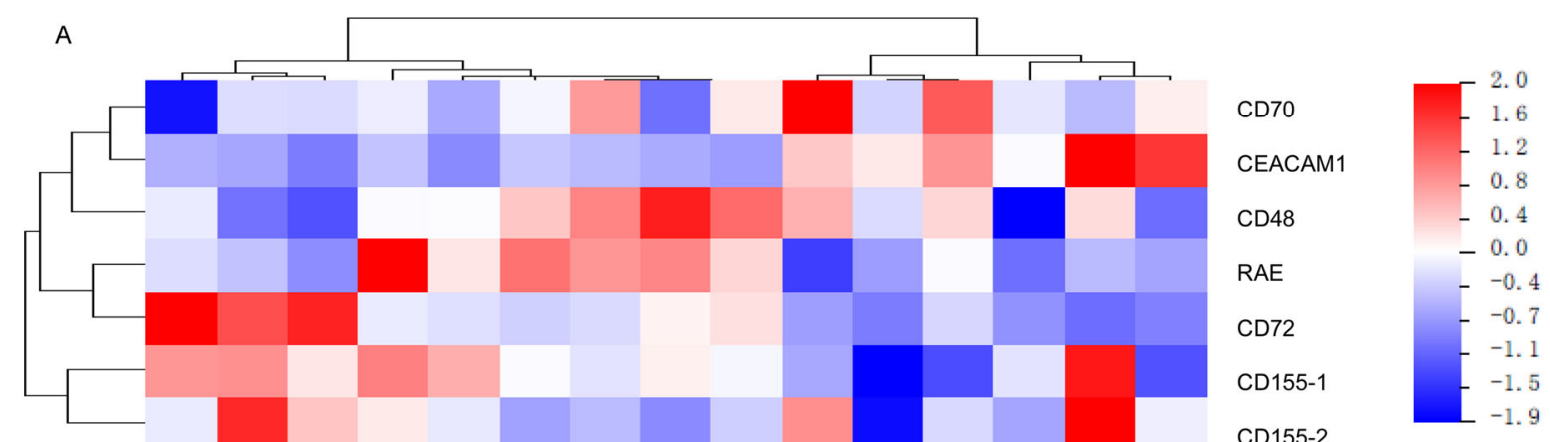

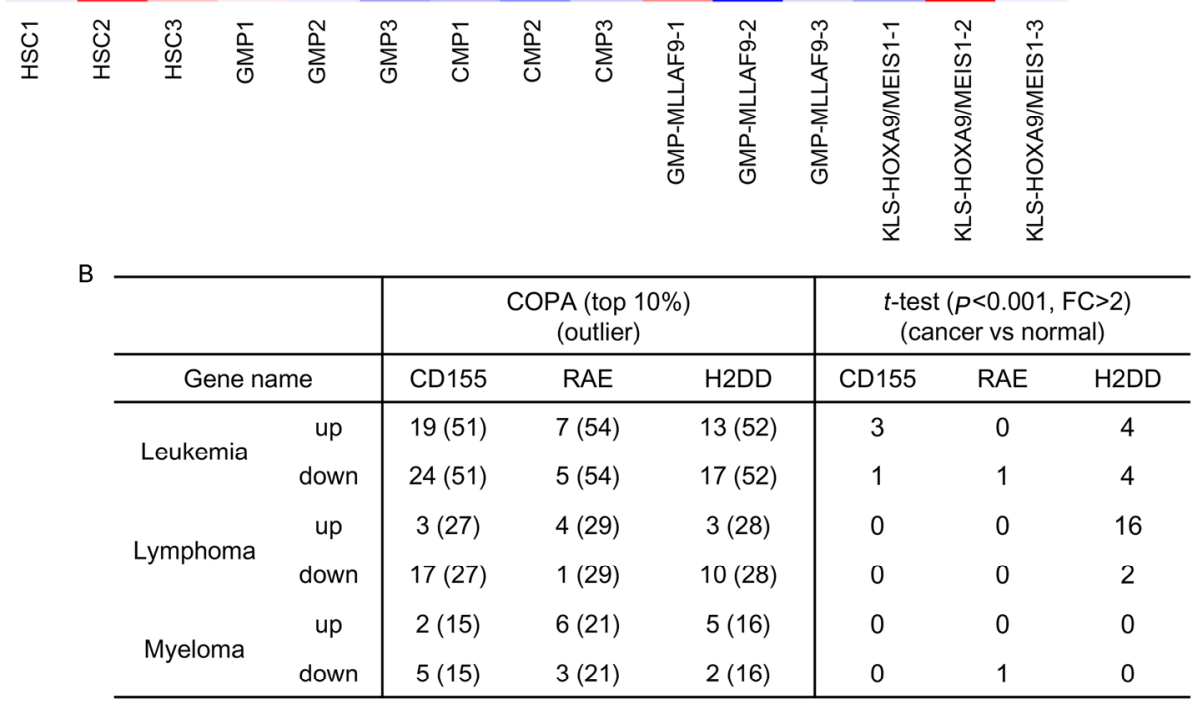

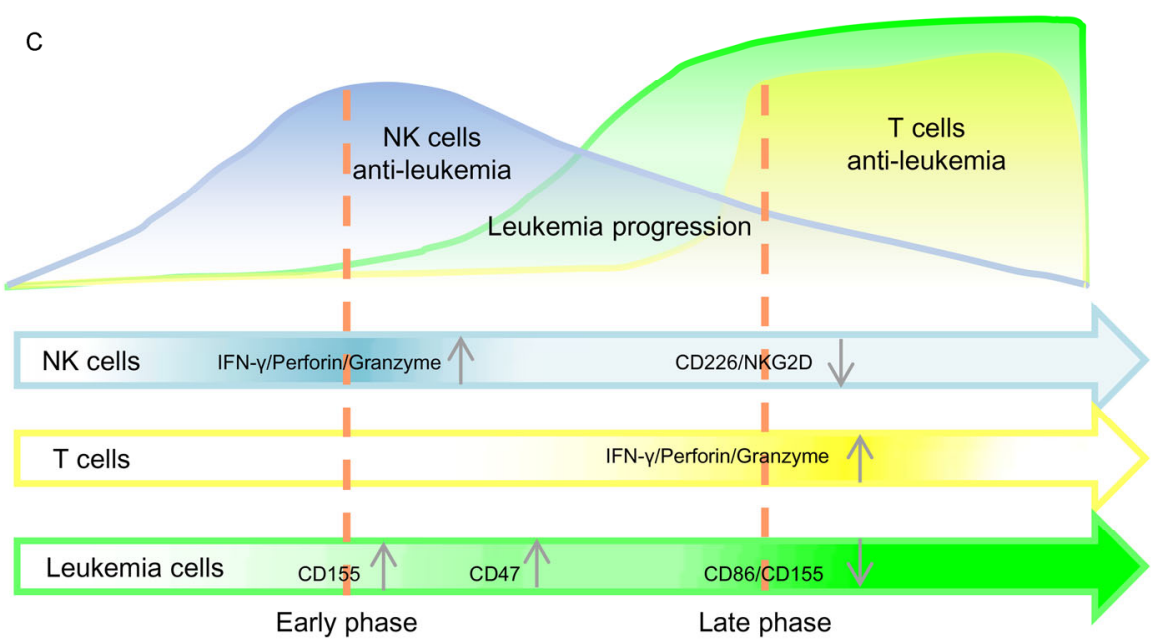

Figure 6 Expression of NK cell-activating ligands on hierarchical leukemic cells. A, Representative heat map of the NK cell-activating ligands in hierarchical hematopoietic cells based on the analysis of reported microarray data (GSE20377). Unsupervised clustering was performed using array tools. B, Summary of selected NK cell-activating ligands in leukemia, lymphoma and myeloma by the Oncomine database. Cancer Outlier Profile Analysis (COPA) and a $t$-test were used to quantify the expression patterns of selected genes. The range and median fractions of patients with selected outlier gene profiles in all datasets are shown. C, Schematic model of the interplay between the immune system and leukemia progression. NK cells perform crucial roles against leukemia in the early phase, whereas T cells attack leukemia in the late phase. Simultaneously, leukemia cells evolve by modifying the expression of immune antigens to escape immunosurveillance.

during the early stage of tumor development. In our study, we clearly demonstrated that NK cells performed anti-leukemia activities during the early stage but were inhibited during the late stage by CD226 and NKG2D downreg- ulation (Figure 5A). Although NKG2D expression was only slightly decreased on day 20, the expression of its ligand RAE was remarkably increased (Figure 5C). NKG2D/RAE might also be involved in leukemia immunosurveillance, 
which was reported in a previous MLL-ENL leukemia model. However, in our model we found the up-regulation of CD155 and the down-regulation of RAE at the initiation of leukemia, indicating that the activation of NK cells in the MLL-AF9 leukemia model occurred mainly through the CD226/CD155 interaction. Nevertheless, the crosstalk between different stimulatory pathways should be kept in mind in future studies. We also observed the upregulation of cytotoxic mole cules in activated NK cells, indicating that NK cells could directly kill malignant cells (Figure 3E). IFN- $\gamma$ was also highly expressed in NK cells, which could in turn induce an adaptive immune response. Although the $\mathrm{T}$ cells in our model were activated during the late stage, for the short latency of our model, the precise crosstalk between NK cells and T cells requires further investigation. CD122 is expressed at a lower level on subsets of T cells, B cells, monocytes and macrophages [35]. However, anti-CD122 antibodies have been used to selectively deplete NK cells in previous studies [27,28]. Because impairment of NK cell cytotoxicity is associated with almost all hematological malignancies, restoration of normal $\mathrm{NK}$ function remains an attractive goal for immunotherapy [16,36].

In conclusion, we found that leukemic cells could survive and expand in hosts with intact immune systems. The leukemic cells escaped from host immunosurveillance via editing their immunogenicity. NK cells were activated in the early phase, which was a necessary step for the elimination of leukemic cells. CD155/CD226 primarily mediated the interaction between leukemic cells and NK cells to achieve the antitumor effects of NK cells during the early phase of AML. The clinical data also showed that CD155 expression was decreased in leukemia. However, the precise mechanisms underlying the involvement of the CD226/CD155 interaction in $\mathrm{AML}$ immunosurveillance require further investigation using antibodies specific to CD155 and some target compounds. Our results demonstrate that NK cells play a significant role in immunosurveillance against leukemic cells during the early stage of AML primarily through the CD226/CD155 interaction; however, NK cells are not sufficient to eliminate the leukemic cells.

The author(s) declare that they have no conflict of interest. All animal experiment in this study were conducted according to the requirements and were maintained in the animal facility of the State Key Institute of Hematology (SKLEH). All experiments were conducted under the protocol approved by the Institutional Animal Care and Use Committees of SKLEH.

We thank Dr. Yayi Hou from Nanjing University for proofreading. We are also grateful to our lab members and collaborators for their assistance with the experiments and in the preparation of the manuscript. This work was supported by grants from the National Natural Science Foundation of China (81421002, 81300374, 81400077, 81300375, 81430004), the Ministry of Science and Technology of China (2011CB964801, 2013CB966902, 2015CB964400), and the General Financial Grant from the China Postdoctoral Science Foundation (2011M500263).
Wang YaJie designed and performed all experiments, analyzed the data and wrote the manuscript. Chen Chen helped with all experiments and assisted with the manuscript. Dong Fang, Ma ShiHui, Zhou Yuan, Cheng Hui and Gong YueMin. helped with the mouse experiments and flow cytometry. Xu Jing helped with the mouse experiment and English editing. Hao Sha conducted research, analyzed the data and assisted with the manuscript. Cheng Tao conceived the study, designed the experiments, interpreted the results, wrote the paper and oversaw the research project.

1 Hope KJ, Jin L, Dick JE. Human acute myeloid leukemia stem cells. Arch Med Res, 2003, 34: 507-514

2 Reinisch A, Chan SM, Thomas D, Majeti R. Biology and clinical relevance of acute myeloid leukemia stem cells. Semin Hematol, 2015, 52: 150-164

3 Buss EC, Ho AD. Leukemia stem cells. Int J Cancer, 2011, 129: 2328-2336

4 Buchner T, Urbanitz D, Ruhl H, Fischer J, Kuse R. Role of chemotherapy for AML in remission. Lancet, 1985, 1: 1224-1225

5 Valcarcel D, Martino R, Caballero D, Martin J, Ferra C, Nieto JB, Sampol A, Bernal MT, Pinana JL, Vazquez L, Ribera JM, Besalduch J, Moraleda JM, Carrera D, Brunet MS, Perez-Simon JA, Sierra J. Sustained remissions of high-risk acute myeloid leukemia and myelodysplastic syndrome after reduced-intensity conditioning allogeneic hematopoietic transplantation: chronic graft-versus-host disease is the strongest factor improving survival. J Clin Oncol, 2008, 26: 577-584

6 Frankel AE, Baer MR, Hogge DE, Stuart RK. Immunotherapy of acute myeloid leukemia. Curr Pharm Biotechnol, 2001, 2: 209-215

7 Lichtenegger FS, Krupka C, Kohnke T, Subklewe M. Immunotherapy for Acute Myeloid Leukemia. Semin Hematol, 2015, 52: 207-214

8 Curran E, Corrales L, Kline J. Targeting the innate immune system as immunotherapy for acute myeloid leukemia. Front Oncol, 2015, 5: 83

9 Anguille S, Van Tendeloo VF, Berneman ZN. Leukemia-associated antigens and their relevance to the immunotherapy of acute myeloid leukemia. Leukemia, 2012, 26: 2186-2196

10 Van Driessche A, Gao L, Stauss HJ, Ponsaerts P, Van Bockstaele DR, Berneman ZN, Van Tendeloo VF. Antigen-specific cellular immunotherapy of leukemia. Leukemia, 2005, 19: 1863-1871

11 Jaiswal S, Jamieson CH, Pang WW, Park CY, Chao MP, Majeti R, Traver D, van Rooijen N, Weissman IL. CD47 is upregulated on circulating hematopoietic stem cells and leukemia cells to avoid phagocytosis. Cell, 2009, 138: 271-285

12 Chen X, Liu S, Wang L, Zhang W, Ji Y, Ma X. Clinical significance of B7-H1 (PD-L1) expression in human acute leukemia. Cancer Biol Ther, 2008, 7: 622-627

13 Spaner DE, Masellis A. Toll-like receptor agonists in the treatment of chronic lymphocytic leukemia. Leukemia, 2007, 21: 53-60

14 de Aquino MT, Malhotra A, Mishra MK, Shanker A. Challenges and future perspectives of T cell immunotherapy in cancer. Immunol Lett, 2015, 166: 117-133

15 Nagorsen D, Scheibenbogen C, Marincola FM, Letsch A, Keilholz U. Natural T cell immunity against cancer. Clin Cancer Res, 2003, 9: 4296-4303

16 Farnault L, Sanchez C, Baier C, Le Treut T, Costello RT. Hematological malignancies escape from NK cell innate immune surveillance: mechanisms and therapeutic implications. Clin Dev Immunol, 2012, 2012: 421702-421710

17 Stringaris K, Sekine T, Khoder A, Alsuliman A, Razzaghi B, Sargeant R, Pavlu J, Brisley G, de Lavallade H, Sarvaria A, Marin D, Mielke S, Apperley JF, Shpall EJ, Barrett AJ, Rezvani K. Leukemia-induced phenotypic and functional defects in natural killer cells predict failure to achieve remission in acute myeloid leukemia. Haematologica, 2014, 99: 836-847

18 Khaznadar Z, Henry G, Setterblad N, Agaugue S, Raffoux E, Boissel N, Dombret H, Toubert A, Dulphy N. Acute myeloid leukemia impairs natural killer cells through the formation of a deficient 
cytotoxic immunological synapse. Eur J Immunol, 2014, 44: 3068-3080

19 Sanchez-Correa B, Campos C, Pera A, Bergua JM, Arcos MJ, Banas H, Casado JG, Morgado S, Duran E, Solana R, Tarazona R. Natural killer cell immunosenescence in acute myeloid leukaemia patients: new targets for immunotherapeutic strategies? Cancer Immunol Immunotherapy, 2015, doi: 10.1007/s00262-015-1720-6

20 Liu Y, Cheng H, Gao S, Lu X, He F, Hu L, Hou D, Zou Z, Li Y, Zhang $\mathrm{H}$, Xu J, Kang L, Wang Q, Yuan W, Cheng T. Reprogramming of MLL-AF9 leukemia cells into pluripotent stem cells. Leukemia, 2014, 28: 1071-1080

21 Cheng H, Hao S, Liu Y, Pang Y, Ma S, Dong F, Xu J, Zheng G, Li S, Yuan W, Cheng T. Leukemic marrow infiltration reveals a novel role for Egr3 as a potent inhibitor of normal hematopoietic stem cell proliferation. Blood, 2015, 126: 1302-1313

22 Shimura N, Kojima S. Effects of low-dose-gamma rays on the immune system of different animal models of disease. Dose Response, 2014, 12: 429-465

23 Nakata J, Nakano K, Okumura A, Mizutani Y, Kinoshita H, Iwai M, Hasegawa K, Morimoto S, Fujiki F, Tatsumi N, Nakajima H, Nakae Y, Nishida S, Tsuboi A, Oji Y, Oka Y, Sugiyama H, Kumanogoh A, Hosen N. In vivo eradication of MLL/ENL leukemia cells by NK cells in the absence of adaptive immunity. Leukemia, 2014, 28 : 1316-1325

24 June CH, Ledbetter JA, Linsley PS, Thompson CB. Role of the CD28 receptor in T-cell activation. Immunol Today, 1990, 11: 211-216

25 Archimbaud E, Thomas X, Campos L, Magaud JP, Dore JF, Fiere D. Expression of surface adhesion molecules CD54 (ICAM-1) and CD58 (LFA-3) in adult acute leukemia: relationship with initial characteristics and prognosis. Leukemia, 1992, 6: 265-271

26 Marotta G, Zagonel V, Pinto A. Induction of LFA-1/CD11a and ICAM-1/CD54 adhesion molecules on neoplastic B cells during in vivo treatment of chronic lymphocytic leukemia with interferon-alpha 2. Blood, 1993, 81: 267-269
27 Tanaka T, Kitamura F, Nagasaka Y, Kuida K, Suwa H, Miyasaka M. Selective long-term elimination of natural killer cells in vivo by an anti-interleukin 2 receptor beta chain monoclonal antibody in mice. $\mathrm{J}$ Exp Med, 1993, 178: 1103-1107

28 McKenzie JL, Gan OI, Doedens M, Dick JE. Human short-term repopulating stem cells are efficiently detected following intrafemoral transplantation into NOD/SCID recipients depleted of CD122+ cells. Blood, 2005, 106: 1259-1261

29 Vivier E, Tomasello E, Baratin M, Walzer T, Ugolini S. Functions of natural killer cells. Nat Immunol, 2008, 9: 503-510

30 Huergo-Zapico L, Acebes-Huerta A, Lopez-Soto A, Villa-Alvarez M, Gonzalez-Rodriguez AP, Gonzalez S. Molecular Bases for the regulation of NKG2D ligands in cancer. Front Immunol, 2014, 5: 106

31 Lopez-Soto A, Huergo-Zapico L, Acebes-Huerta A, Villa-Alvarez M, Gonzalez S. NKG2D signaling in cancer immunosurveillance. Int J Cancer, 2015, 136: 1741-1750

32 Passegue E, Jamieson CH, Ailles LE, Weissman IL. Normal and leukemic hematopoiesis: are leukemias a stem cell disorder or a reacquisition of stem cell characteristics? Proc Natl Acad Sci USA, 2003, 100 Suppl 1: 11842-11849

33 Sungur CM, Murphy WJ. Positive and negative regulation by NK cells in cancer. Crit Rev Oncog, 2014, 19: 57-66

34 Bottino C, Dondero A, Bellora F, Moretta L, Locatelli F, Pistoia V, Moretta A, Castriconi R. Natural killer cells and neuroblastoma: tumor recognition, escape mechanisms, and possible novel immunotherapeutic approaches. Front Immunol, 2014, 5: 56

35 Taussig DC, Miraki-Moud F, Anjos-Afonso F, Pearce DJ, Allen K, Ridler C, Lillington D, Oakervee H, Cavenagh J, Agrawal SG, Lister TA, Gribben JG, Bonnet D. Anti-CD38 antibody-mediated clearance of human repopulating cells masks the heterogeneity of leukemia-initiating cells. Blood, 2008, 112: 568-575

36 Pittari G, Filippini P, Gentilcore G, Grivel JC, Rutella S. Revving up natural killer cells and cytokine-induced killer cells against hematological malignancies. Front Immunol, 2015, 6: 230

Open Access This article is distributed under the terms of the Creative Commons Attribution License which permits any use, distribution, and reproduction in any medium, provided the original author(s) and source are credited. 